\title{
Aza-Morita-Baylis-Hillman reaction of maleimides with azodicarboxylates under neat conditions
}

\author{
SUBRAMANI KANDHASAMY, KESAVAN KARTHIKEYAN, \\ KRISHNAN RAMACHANDIRAN, DORAISWAMY MURALIDHARAN and \\ PARAMASIVAN THIRUMALAI PERUMAL* \\ Organic Chemistry Division, CSIR-Central Leather Research Institute, Adyar, Chennai, 600 020, India \\ e-mail: ptperumal@gmail.com
}

MS received 12 December 2012; revised 2 March 2013; accepted 5 April 2013

\begin{abstract}
Green approach on reaction of diisopropylazodicarboxylate or diethyl azodicarboxylate with maleimides proceeded smoothly under neat condition at $70^{\circ} \mathrm{C}$ to give the corresponding hydrazine substituted pyrrolidinone as aza-Morita-Baylis-Hillman adducts in moderate to good yields in the presence of 1,4-diazabicyclo[2.2.2] octane (DABCO) as nitrogen Lewis base.
\end{abstract}

Keywords. Aza-Morita-Baylis-Hillman reaction; pyrrolidone; azo compound; neat reaction.

\section{Introduction}

The Morita-Baylis-Hillman (MBH) reaction, one of the most atom economical and important carboncarbon bond-forming reactions, ${ }^{1,2}$ has made great progress recently in the areas of shortening reaction time, extending the scope of the substrates, asymmetric catalysis and mechanistic studies. ${ }^{3-5}$ For its great potential of the products for further transformation and the superior mild reaction conditions, the aza version of this reaction is extremely fascinating. ${ }^{6-8}$ Recently, the aza-MBH reaction has received much attention $^{9}$ in which $N$-sulphonated imines $(\mathrm{ArCH}=\mathrm{NTs})$ and $N$-phosphorated imines $\left[\mathrm{ArCH}=\mathrm{NP}(\mathrm{O}) \mathrm{R}^{2}\right]$ were employed as electrophiles. Reports on the usage of diisopropylazodicarboxylate (DIAD) and diethyl azodicarboxylate (DEAD) as electrophiles are scanty. ${ }^{10}$ Over the past few years, organic reactions under solvent-free conditions have gained much popularity. ${ }^{11}$ Solvent-free reaction conditions are not only of interest from ecological point of view, but in many cases, also offer several synthetic advantages like higher yields, reduced pollution, low cost, improved selectivities, and simplicity in process and handling. ${ }^{12}$

The substituted pyrrole ring system ${ }^{13}$ is the basic building block of a number of important biological compounds including vitamin B12, chlorophyll, heme,

*For correspondence etc. ${ }^{14-16}$ Similarly, substituted pyrrolidinones are important components in the preparation of bile pigments, ${ }^{17}$ and of several classes of bioactive natural compounds with a wide range of activity. ${ }^{13(b), 18}$ Figure 1 shows some of the pyrrole-based bioactive natural products.

During the course of our investigations, we found that maleimides act as activated alkenes in the $\mathrm{MBH}$ reaction to synthesize spirobenzofuranols. ${ }^{18}$ In continuation of our interest in the synthesis of heterocycles ${ }^{19}$ and the $\mathrm{MBH}$ reaction, ${ }^{1(\mathrm{~g}), 20,21}$ we report here aza-MBH reaction of DIAD or DEAD with maleimides in the presence of different Lewis bases and solvents.

\section{Experimental}

Melting points are uncorrected. IR spectra were recorded on a Perkin Elmer FT-IR spectrophotometer. ${ }^{1} \mathrm{H}$ and ${ }^{13} \mathrm{C}$ NMR spectra were recorded in $\mathrm{CDCl}_{3}$ using TMS as an internal standard on a JEOL spectrometer at $500 \mathrm{MHz}$ and $125 \mathrm{MHz}$, respectively. Mass spectra were recorded on a Thermo Finnigan LCQ Advantage MAX 6000 ESI spectrometer. Elemental analyses were recorded using a Thermo Finnigan FLASH EA $1112 \mathrm{CHN}$ analyzer. Column chromatography was performed on silica gel (100-200 mesh, SRL, India). Analytical TLC was performed on precoated plastic sheets of silica gel G/UV-254 of $0.2 \mathrm{~mm}$ thickness (Merck, Germany). 


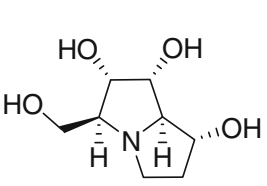

2,3,7-Triepiaustraline

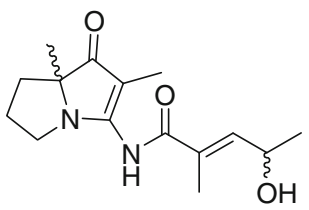

Jenamidines A1/A2

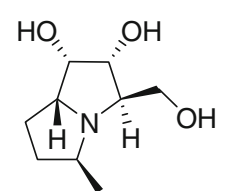

Hyacinthacine A7

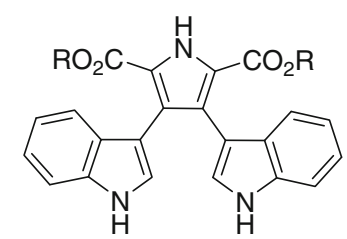

Lycogarubin C
Figure 1. Natural products containing pyrrole moiety.

\subsection{Experimental procedure for the synthesis} of aza-MBH adducts $\mathbf{3} \boldsymbol{a}-\mathbf{j}$

A mixture of diisopropyl or diethyl azodicarboxylate 1a,b (1 mmol, 1.0 equiv.), maleimides $\mathbf{2 a - e}(1 \mathrm{mmol}$, 1.0 equiv.) and DABCO (20 mol \%) was stirred at $70^{\circ} \mathrm{C}$ under neat condition for $2-6 \mathrm{~h}$. The residue was dissolved in ethyl acetate $(20 \mathrm{~mL})$ and given a dil. $\mathrm{HCl}$ wash $(1 \times 20 \mathrm{ml})$ and water wash $(2 \times 20 \mathrm{~mL})$. The ethyl acetate layer was dried over anhydrous $\mathrm{Na}_{2} \mathrm{SO}_{4}$ and removal of solvent under reduced pressure gave a crude product, which was purified by flash column chromatography with ethyl acetate: petroleum ether (20:80) as a elutent to obtain aza-MBH adducts $\mathbf{3 a}-\mathbf{j}$.

2.1a Diisopropyl-1-(1-methyl-2,5-dioxo-2,5-dihydro1 H-pyrrol-3-yl)hydrazine-1,2-dicarboxylate (3a): Pale yellow; viscous liquid; IR $\left(\mathrm{CH}_{2} \mathrm{Cl}_{2}\right)$ : 3445 , 2991, 1725, 1695, 1622, 1443, $1154 \mathrm{~cm}^{-1} ;{ }^{1} \mathrm{H}$ NMR $\left(500 \mathrm{MHz}, \mathrm{CDCl}_{3}\right): \delta 1.30-1.33(\mathrm{~m}, 12 \mathrm{H}), 2.97(\mathrm{~s}$, 3H), 4.96-5.07 (m, 2H), 6.48 (s, 1H) 7.10 (brs, 1H); ${ }^{13} \mathrm{C} \mathrm{NMR}\left(125 \mathrm{MHz}, \mathrm{CDCl}_{3}\right): \delta 21.7,21.8,21.9,22.0$, $23.8,70.1,73.1,110.7,141.3,152.9,170.0 ; \mathrm{MS} \mathrm{m} / \mathrm{z}=$ $314[\mathrm{M}+\mathrm{H}]^{+}$; Anal. Calcd for $\mathrm{C}_{13} \mathrm{H}_{19} \mathrm{~N}_{3} \mathrm{O}_{6}$ (313.13): C, 49.84; H, 6.11; N, 13.41. Found: C, 49.72; H, 6.13; N, 13.36.

2.1b Diisopropyl-1-(2,5-dioxo-1-phenyl-2,5-dihydro$1 \mathrm{H}$-pyrrol-3-yl)hydrazine-1,2-dicarboxylate (3b): Pale yellow; viscous liquid; IR $\left(\mathrm{CH}_{2} \mathrm{Cl}_{2}\right)$ : 3448, 2987, 1729, 1679, 1626, 1442, $1158 \mathrm{~cm}^{-1} ;{ }^{1} \mathrm{H}$ NMR $\left(500 \mathrm{MHz}, \mathrm{CDCl}_{3}\right): \delta 1.30-1.35(\mathrm{~m}, 12 \mathrm{H}), 4.96-5.11$ $(\mathrm{m}, 2 \mathrm{H}), 6.59(\mathrm{~s}, 1 \mathrm{H}), 7.11$ (brs, 1H), $7.31(\mathrm{~d}, 2 \mathrm{H}$, $J=6.9 \mathrm{~Hz}), 7.35(\mathrm{t}, 1 \mathrm{H}, J=7.6 \mathrm{~Hz}), 7.44(\mathrm{t}, 2 \mathrm{H}$, $J=7.6 \mathrm{~Hz}) ;{ }^{13} \mathrm{C} \mathrm{NMR}\left(125 \mathrm{MHz}, \mathrm{CDCl}_{3}\right): \delta 21.8$, $21.9,22.0,71.0,73.4,111.2,126.4,128.1,129.2$, $131.1,142.1,152.6,168.7 ; \mathrm{MS} \mathrm{m} / \mathrm{z}=376[\mathrm{M}+\mathrm{H}]^{+}$;
Anal. Calcd for $\mathrm{C}_{18} \mathrm{H}_{21} \mathrm{~N}_{3} \mathrm{O}_{6}$ (375.14): C, 57.59; H, 5.64; N, 11.19. Found: C, 57.81; H, 5.66; N, 11.12.

2.1c Diisopropyl-1-(2,5-dioxo-2,5-dihydro-1H-pyrrol3-yl)hydrazine-1,2-dicarboxylate (3c): Colourless viscous liquid; IR $\left(\mathrm{CH}_{2} \mathrm{Cl}_{2}\right): 3439,2996,1719,1675$, 1629, 1443, $1158 \mathrm{~cm}^{-1} ;{ }^{1} \mathrm{H}$ NMR $\left(500 \mathrm{MHz}, \mathrm{CDCl}_{3}\right)$ : ठ 1.25-1.32 (m, 12H), 4.96-5.07 (m, 2H), $6.48(\mathrm{~s}, 1 \mathrm{H})$, 7.17 (brs, 1H), 7.67 (brs, 1H); ${ }^{13} \mathrm{C}$ NMR $(125 \mathrm{MHz}$, $\left.\mathrm{CDCl}_{3}\right): \delta 21.8,21.9,70.9,73.3,111.8,141.9,152.9$, 169.4; MS $\mathrm{m} / \mathrm{z}=300[\mathrm{M}+\mathrm{H}]^{+}$; Anal. Calcd for $\mathrm{C}_{12} \mathrm{H}_{17} \mathrm{~N}_{3} \mathrm{O}_{6}$ (299.11): C, 48.16; H, 5.73; N, 14.04 . Found: C, 48.23; H, 5.68; N, 14.01.

2.1d Diisopropyl-1-(2,5-dioxo-1-p-tolyl-2,5-dihydro$1 \mathrm{H}$-pyrrol-3-yl)hydrazine-1,2-dicarboxylate $\quad(3 \mathrm{~d})$ : Yellow solid; mp $128-130^{\circ} \mathrm{C}$; IR (KBr): 3442, 2997 , 1728, 1685, 1621, 1448, $1157 \mathrm{~cm}^{-1}$; ${ }^{1} \mathrm{H}$ NMR $\left(500 \mathrm{MHz}, \mathrm{CDCl}_{3}\right): \delta 1.24-1.32(\mathrm{~m}, 12 \mathrm{H}), 2.34(\mathrm{~s}$, $3 \mathrm{H}), 4.95-5.07(\mathrm{~m}, 2 \mathrm{H}), 6.62(\mathrm{~s}, 1 \mathrm{H}), 7.15(\mathrm{~d}, 2 \mathrm{H}$, $J=8.4 \mathrm{~Hz}), 7.21(\mathrm{~d}, 2 \mathrm{H}, J=8.4 \mathrm{~Hz}), 7.36$ (brs, $1 \mathrm{H})$; ${ }^{13} \mathrm{C} \mathrm{NMR}\left(125 \mathrm{MHz}, \mathrm{CDCl}_{3}\right): \delta 21.2,21.7,21.8,21.9$, 70.9, 73.2, 110.9, 126.3, 128.5, 129.8, 138.1, 141.5, 152.9, 168.9; MS m/z $=390[\mathrm{M}+\mathrm{H}]^{+}$; Anal. Calcd for $\mathrm{C}_{19} \mathrm{H}_{23} \mathrm{~N}_{3} \mathrm{O}_{6}$ (389.16): C, 58.60; H, 5.95; N, 10.79 . Found: C, 58.42; H, 5.96; N, 10.81 .

2.1e Diisopropyl-1-(1-(4-bromophenyl)-2,5-dioxo-2,5dihydro-1 H-pyrrol-3-yl)hydrazine-1,2-dicarboxylate $(3 \boldsymbol{e})$ : Colourless viscous liquid; IR $\left(\mathrm{CH}_{2} \mathrm{Cl}_{2}\right): 3441$, 2998, 1725, 1685, 1622, 1449, $1155 \mathrm{~cm}^{-1} ;{ }^{1} \mathrm{H}$ NMR $\left(500 \mathrm{MHz}, \mathrm{CDCl}_{3}\right): \delta 1.29-1.33(\mathrm{~m}, 12 \mathrm{H}), 4.96-5.09$ $(\mathrm{m}, 2 \mathrm{H}), 6.65(\mathrm{~s}, 1 \mathrm{H}), 7.19-7.21(\mathrm{~m}, 2 \mathrm{H}), 7.55(\mathrm{~d}$, $2 \mathrm{H}, J=8.4 \mathrm{~Hz}) ;{ }^{13} \mathrm{C} \mathrm{NMR}\left(125 \mathrm{MHz}, \mathrm{CDCl}_{3}\right): \delta$ 21.8, 21.9, 22.0, 71.0, 73.4, 111.1, 121.7, 127.7, 130.2, 132.3, 141.6, 152.9, 168.3; MS m/z $=454[\mathrm{M}+\mathrm{H}]^{+}$; Anal. Calcd for $\mathrm{C}_{18} \mathrm{H}_{20} \mathrm{BrN}_{3} \mathrm{O}_{6}$ (453.05): $\mathrm{C}$, 47.59; $\mathrm{H}$, 4.44; N, 9.25. Found: C, 47.66; H, 4.47; N, 9.39.

2.1f Diethyl-1-(1-methyl-2,5-dioxo-2,5-dihydro- $1 \mathrm{H}$ pyrrol-3-yl)hydrazine-1,2-dicarboxylate (3f): Pale yellow; viscous liquid; IR $\left(\mathrm{CH}_{2} \mathrm{Cl}_{2}\right): 3431,2997,1755$, 1694, 1622, 1445, $1157 \mathrm{~cm}^{-1} ;{ }^{1} \mathrm{H}$ NMR $(500 \mathrm{MHz}$, $\left.\mathrm{CDCl}_{3}\right): \delta 1.29(\mathrm{t}, 3 \mathrm{H}, J=7.6 \mathrm{~Hz}), 1.33(\mathrm{t}, 3 \mathrm{H}$, $J=6.9 \mathrm{~Hz}), 2.97(\mathrm{~s}, 3 \mathrm{H}), 4.21(\mathrm{q}, 2 \mathrm{H}, J=6.9 \mathrm{~Hz})$, $4.30(\mathrm{q}, 2 \mathrm{H}, J=7.6 \mathrm{~Hz}), 6.73(\mathrm{~s}, 1 \mathrm{H}), 7.16$ (brs, $1 \mathrm{H}) ;{ }^{13} \mathrm{C}$ NMR $\left(125 \mathrm{MHz}, \mathrm{CDCl}_{3}\right): \delta 14.2 .14 .3,23.8$, $62.9,64.7,110.9,141.4,153.4,169.9 ; \mathrm{MS} \mathrm{m} / \mathrm{z}=286$ $[\mathrm{M}+\mathrm{H}]^{+}$; Anal. Calcd for $\mathrm{C}_{11} \mathrm{H}_{15} \mathrm{~N}_{3} \mathrm{O}_{6}$ (285.10): C, 46.32; H, 5.30; N, 14.73. Found: C, 46.19; H, 5.32; N, 14.81 . 
2.1g Diethyl-1-(2,5-dioxo-1-phenyl-2,5-dihydro-1 Hpyrrol-3-yl)hydrazine-1,2-dicarboxylate (3g): Pale yellow; viscous liquid; IR $\left(\mathrm{CH}_{2} \mathrm{Cl}_{2}\right): 3428,2994,1751$, $1695,1621,1446,1159 \mathrm{~cm}^{-1} ;{ }^{1} \mathrm{H}$ NMR $(500 \mathrm{MHz}$, $\left.\mathrm{CDCl}_{3}\right): \delta 1.27(\mathrm{t}, 3 \mathrm{H}, J=7.6 \mathrm{~Hz}), 1.34(\mathrm{t}, 3 \mathrm{H}$, $J=7.6 \mathrm{~Hz}), 4.22(\mathrm{q}, 2 \mathrm{H}, J=7.6 \mathrm{~Hz}), 4.34(\mathrm{q}, 2 \mathrm{H}$, $J=7.6 \mathrm{~Hz}), 6.67(\mathrm{~s}, 1 \mathrm{H}), 7.29(\mathrm{~d}, 2 \mathrm{H}, J=7.6 \mathrm{~Hz})$, $7.34(\mathrm{t}, 1 \mathrm{H}, J=7.6 \mathrm{~Hz}), 7.41-7.44(\mathrm{~m}, 3 \mathrm{H}) ;{ }^{13} \mathrm{C} \mathrm{NMR}$ $\left(125 \mathrm{MHz}, \mathrm{CDCl}_{3}\right): \delta 14.3,14.5,62.9,64.8,111.3$, $126.3,128.1,129.2,131.1,141.2,153.4,168.6$; MS $\mathrm{m} / \mathrm{z}=348[\mathrm{M}+\mathrm{H}]^{+}$; Anal. Calcd for $\mathrm{C}_{16} \mathrm{H}_{17} \mathrm{~N}_{3} \mathrm{O}_{6}$ (347.11): C, 55.33; H, 4.93; N, 12.10. Found: C, 55.42; H, 4.89; N, 12.19.

2.1h Diethyl-1-(2,5-dioxo-2,5-dihydro-1 H-pyrrol-3yl)hydrazine-1,2-dicarboxylate (3h): Yellow solid; mp 135-137 ${ }^{\circ}$; IR (KBr): 3435, 2996, 1754, 1692, $1622,1445,1159 \mathrm{~cm}^{-1} ;{ }^{1} \mathrm{H}$ NMR $\left(500 \mathrm{MHz}, \mathrm{CDCl}_{3}\right)$ : $\delta 1.29(\mathrm{t}, 3 \mathrm{H}, J=7.6 \mathrm{~Hz}), 1.34(\mathrm{t}, 3 \mathrm{H}, J=6.9 \mathrm{~Hz})$, $4.23(\mathrm{q}, 2 \mathrm{H}, J=6.9 \mathrm{~Hz}), 4.34(\mathrm{q}, 2 \mathrm{H}, J=6.9 \mathrm{~Hz})$, $6.52(\mathrm{~s}, 1 \mathrm{H}), 7.23$ (brs, $1 \mathrm{H}), 7.44$ (brs, $1 \mathrm{H}) ;{ }^{13} \mathrm{C} \mathrm{NMR}$ $\left(125 \mathrm{MHz}, \mathrm{CDCl}_{3}\right): \delta$ 14.2. 14.8, 62.7, 65.2, 110.4, 141.7, 153.6, 167.2; MS m/z $=272[\mathrm{M}+\mathrm{H}]^{+}$; Anal. Calcd for $\mathrm{C}_{10} \mathrm{H}_{13} \mathrm{~N}_{3} \mathrm{O}_{6}$ (271.08): C, 44.28; H, 4.83; N, 15.49. Found: C, 44.37; H, 4.82; N, 15.55 . 2.1i Diethyl-1-(2,5-dioxo-1- p-tolyl-2,5-dihydro- $1 \mathrm{H}$ pyrrol-3-yl)hydrazine-1,2-dicarboxylate (3i): Colour less solid; mp 132-134 ${ }^{\circ} \mathrm{C}$; IR (KBr): 3432, 2995, 1752, 1694, 1622, 1441, $1159 \mathrm{~cm}^{-1} ;{ }^{1} \mathrm{H}$ NMR $(500 \mathrm{MHz}$, $\left.\mathrm{CDCl}_{3}\right): \delta 1.27(\mathrm{t}, 3 \mathrm{H}, J=7.6 \mathrm{~Hz}), 1.34(\mathrm{t}, 3 \mathrm{H}$, $J=6.9 \mathrm{~Hz}), 2.35(\mathrm{~s}, 3 \mathrm{H}), 4.22(\mathrm{q}, 2 \mathrm{H}, J=7.6 \mathrm{~Hz})$, $4.35(\mathrm{q}, 2 \mathrm{H}, J=6.9 \mathrm{~Hz}), 6.65(\mathrm{~s}, 1 \mathrm{H}), 7.16(\mathrm{~d}, 2 \mathrm{H}$, $J=8.4 \mathrm{~Hz}), 7.23(\mathrm{~d}, 2 \mathrm{H}, J=7.7 \mathrm{~Hz}), 7.40$ (brs, $1 \mathrm{H}) ;{ }^{13} \mathrm{C} \mathrm{NMR}\left(125 \mathrm{MHz}, \mathrm{CDCl}_{3}\right): \delta 14.3,14.5,21.2$, $62.9,64.8,111.3,126.3,128.3,129.8,138.2,141.1$, 153.4, 168.8; MS m/z $=362[\mathrm{M}+\mathrm{H}]^{+}$; Anal. Calcd for $\mathrm{C}_{17} \mathrm{H}_{19} \mathrm{~N}_{3} \mathrm{O}_{6}$ (361.13): C, 56.51; $\mathrm{H}, 5.30 ; \mathrm{N}, 11.63$. Found: C, 56.42; H, 5.32; N, 11.54 .

2.1j Diethyl-1-(1-(4-bromophenyl)-2,5-dioxo-2,5dihydro-1 H-pyrrol-3-yl)hydrazine-1,2-dicarboxylate (3j): Pale yellow; viscous liquid; IR $\left(\mathrm{CH}_{2} \mathrm{Cl}_{2}\right): 3432$, 2997, 1762, 1696, 1622, 1447, $1159 \mathrm{~cm}^{-1} ;{ }^{1} \mathrm{H}$ NMR $\left(500 \mathrm{MHz}, \mathrm{CDCl}_{3}\right): \delta 1.35(\mathrm{t}, 3 \mathrm{H}, J=7.6 \mathrm{~Hz}), 1.49(\mathrm{t}$, $3 \mathrm{H}, J=6.9 \mathrm{~Hz}), 4.12(\mathrm{q}, 2 \mathrm{H}, J=7.6 \mathrm{~Hz}), 4.37(\mathrm{q}, 2 \mathrm{H}$, $J=6.9 \mathrm{~Hz}), 6.68(\mathrm{~s}, 1 \mathrm{H}), 7.20(\mathrm{~d}, 2 \mathrm{H}, J=7.6 \mathrm{~Hz})$, $7.22(\mathrm{brs}, 1 \mathrm{H}), 7.56(\mathrm{~d}, 2 \mathrm{H}, J=7.6 \mathrm{~Hz}) ;{ }^{13} \mathrm{C} \mathrm{NMR}$ $\left(125 \mathrm{MHz}, \mathrm{CDCl}_{3}\right): \delta 14.3,14.5,63.1,64.9,111.3$, 119.6, 124.7, 127.7, 132.3, 141.3, 151.9, 168.1; MS $\mathrm{m} / \mathrm{z}=426[\mathrm{M}+\mathrm{H}]^{+}$; Anal. Calcd for $\mathrm{C}_{16} \mathrm{H}_{16} \mathrm{BrN}_{3} \mathrm{O}_{6}$

Table 1. The effects of Lewis base and solvent in the aza-MBH reaction of DIAD 1a with $N$-methyl maleimide 2a forming $\mathbf{3 a}$.

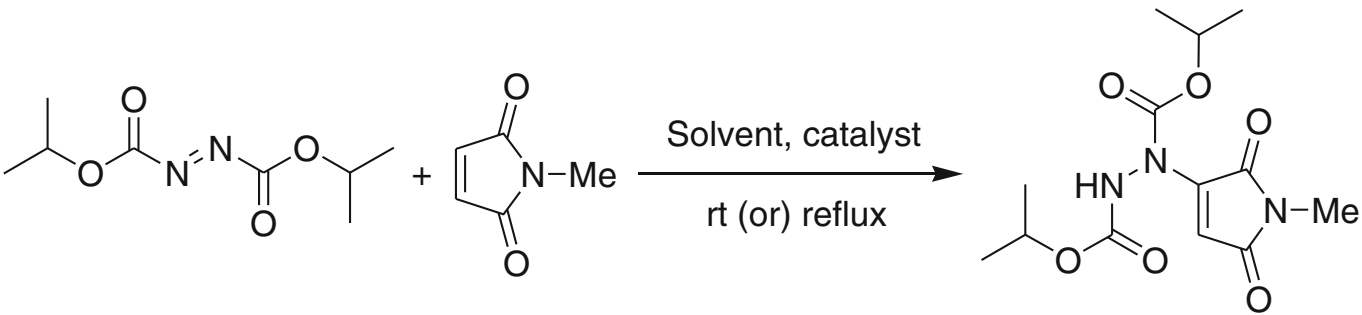

$1 a$

$2 a$

$3 a$

\begin{tabular}{|c|c|c|c|c|c|c|}
\hline Entry & Solvent & Catalyst & $\mathrm{mol} \%$ & Product & Time (h) & Yield $\%^{\mathrm{a}}$ \\
\hline 1 & $\mathrm{MeOH}, \mathrm{rt}$ & $\mathrm{PPh}_{3}$ & 20 & No reaction & - & - \\
\hline 2 & $\mathrm{MeOH}, \mathrm{rt}$ & DABCO & 20 & $3 \mathbf{a}$ & 10 & 25 \\
\hline 3 & $\mathrm{MeOH}$, reflux & DABCO & 20 & $3 a$ & 6 & 40 \\
\hline 4 & $\mathrm{CH}_{2} \mathrm{Cl}_{2}, \mathrm{rt}$ & DABCO & 20 & 3a & 12 & 28 \\
\hline 5 & THF, $\mathrm{rt}$ & DABCO & 20 & 3a & 5 & 50 \\
\hline 6 & $\mathrm{DMF}, \mathrm{rt}$ & DABCO & 20 & 3a & 6 & 60 \\
\hline 7 & Neat, $70^{\circ} \mathrm{C}$ & DABCO & 20 & 3a & 4 & 85 \\
\hline 8 & Neat, $70^{\circ} \mathrm{C}$ & DMAP & 20 & 3a & 8 & 65 \\
\hline 9 & Neat, $70^{\circ} \mathrm{C}$ & $\mathrm{Et}_{3} \mathrm{~N}$ & 20 & 3a & 8 & 52 \\
\hline 10 & Neat, $70^{\circ} \mathrm{C}$ & DBU & 20 & 3a & 5 & 58 \\
\hline
\end{tabular}

${ }^{\mathrm{a}}$ Isolated yield after column chromatography 
Table 2. Aza-MBH reaction of azodicarboxylate $\mathbf{1 a}, \mathbf{b}$ with maleimides $\mathbf{2 a - e}$.

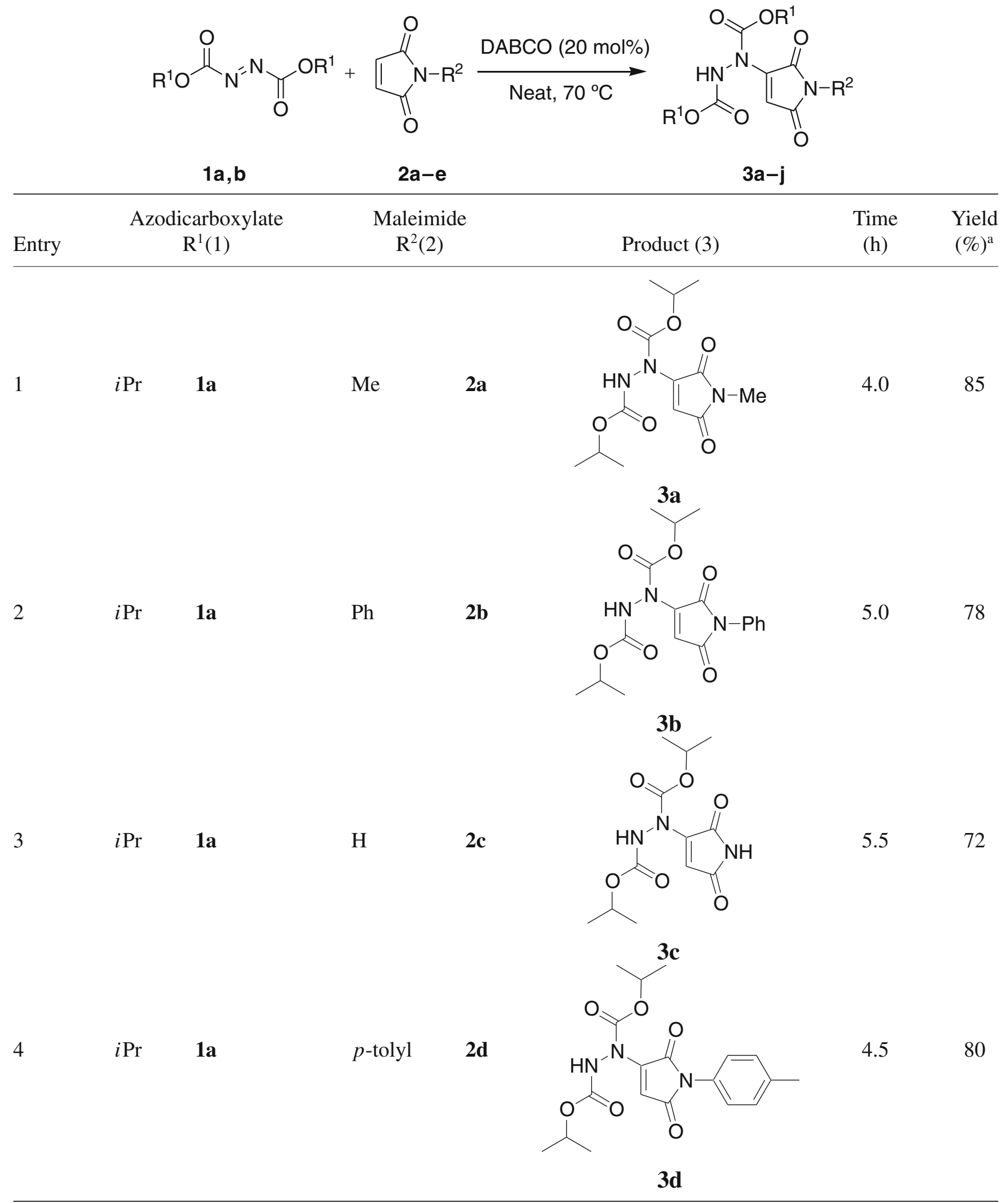


Table 2. (Continued).

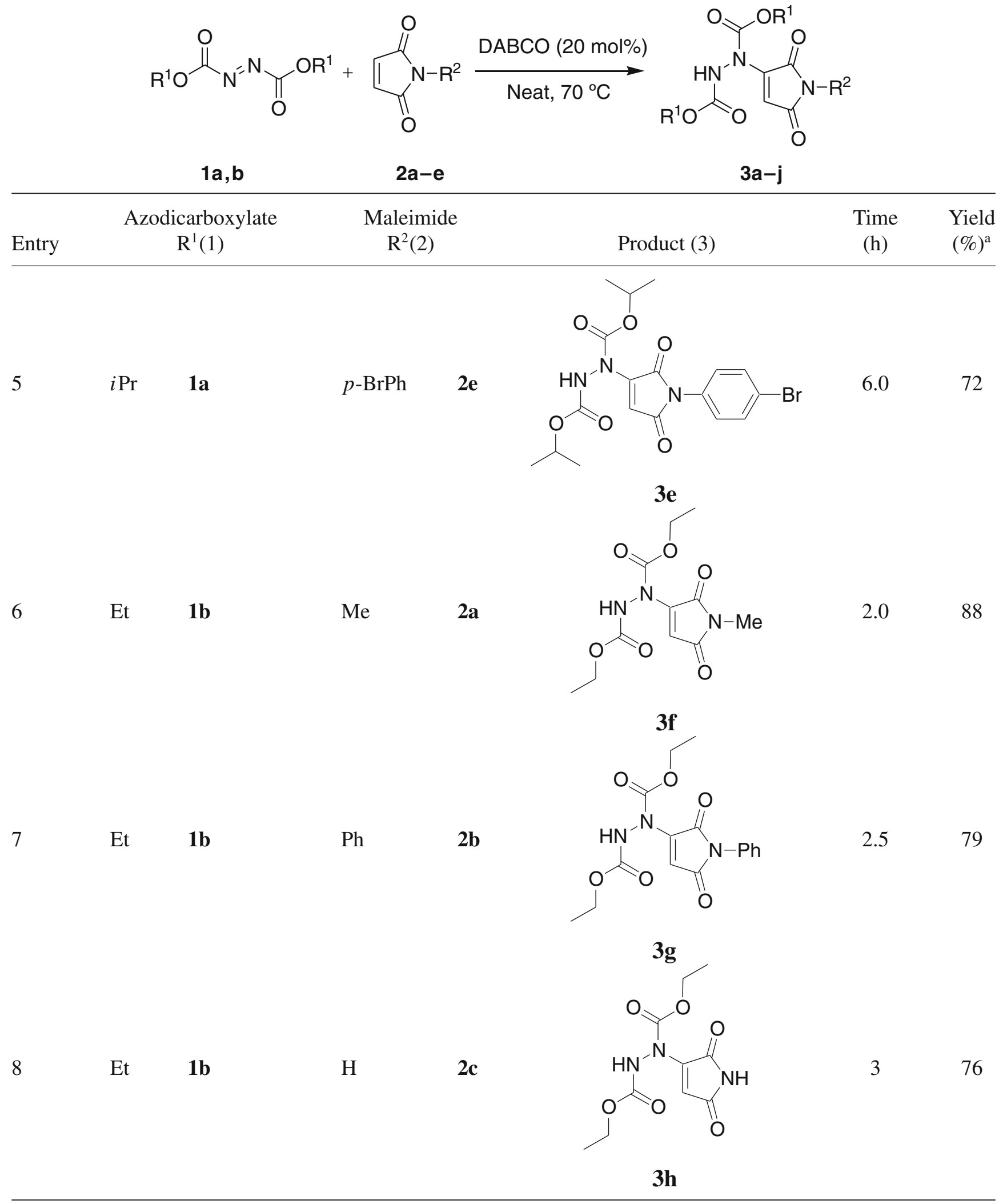


Table 2. (Continued).

Entry

${ }^{a}$ Isolated yield after column chromatography

(425.02): C, 45.09; H, 3.78; N, 9.86. Found: C, 45.24; $\mathrm{H}, 3.80 ; \mathrm{N}, 9.81$.

\section{Results and discussion}

Different catalysts were first examined by carrying out the reaction of DIAD 1a with $N$-methyl maleimide $\mathbf{2 a}$ in $\mathrm{MeOH}$ at room temperature. The results are summarized in table 1. Phosphane Lewis base such as triphenylphosphine $\left(\mathrm{PPh}_{3}\right)$ showed no catalytic activity for this reaction (table 1 , entry 1 ). Nitrogen Lewis base such as DABCO $(20 \mathrm{~mol} \%)$ (table 1 , entry 2$)$ could serve as an effective catalyst to give the corresponding adduct 3a, while the yield was only $25 \%$. Increase in temperature moderately increased the yield to $40 \%$ after $6 \mathrm{~h}$ (table 1, entry 3 ). There was no change in the yield with the increase in the catalytic amount of DABCO. Using $\mathrm{DABCO}$ as the catalyst, the reaction conditions were further optimized with various solvents such as
$\mathrm{CH}_{2} \mathrm{Cl}_{2}$, THF, DMF at room temperature. The yield further increased to $28 \%, 50 \%$ and $60 \%$, respectively (table 1, entries 4-6). On the other hand, under neat condition at $70^{\circ} \mathrm{C}$ the adduct $\mathbf{3 a}$ was obtained in $85 \%$ yield (table 1, entry 7). Its structure was determined by spectroscopic data and elemental analysis.

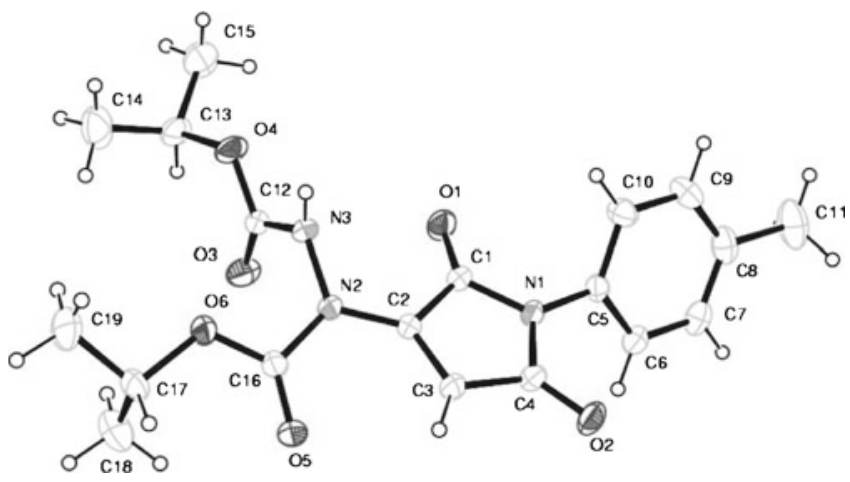

Figure 2. ORTEP diagram of compound $\mathbf{3 d}$. 

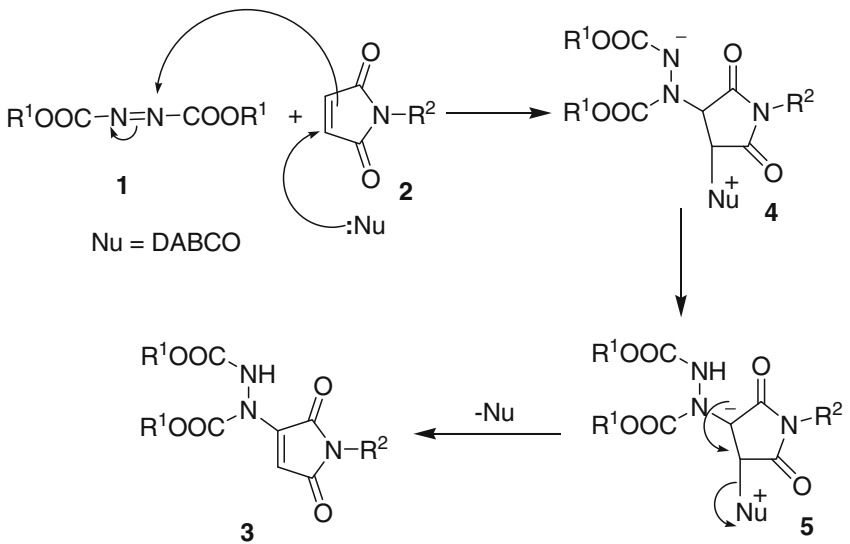

Figure 3. Plausible reaction mechanism.

In the ${ }^{1} \mathrm{H}$ NMR spectrum of compound 3a, the three protons singlet at $\delta 2.97 \mathrm{ppm}$ was attributed to $N-$ $\mathrm{CH}_{3}$ group. The two singlets at $\delta 6.48$ and $7.10 \mathrm{ppm}$ were assigned to the olefinic and $-\mathrm{NH}$ protons, respectively. The latter signal disappeared on $\mathrm{D}_{2} \mathrm{O}$ exchange. In ${ }^{13} \mathrm{C}$ NMR spectrum, peaks at $\delta 152.9$ and $170.0 \mathrm{ppm}$ were assigned to carbamate carbonyl and amide carbons respectively. The mass spectrum revealed the molecular ion peak $[\mathrm{M}+\mathrm{H}]^{+}$at $m / z 314$.

The best reaction condition for this type of aza-MBH reaction is to carry out the reaction under neat condition using DABCO as a Lewis base at $70^{\circ} \mathrm{C}$. Under the optimized reaction condition the aza-MBH reaction of DIAD (1a) or DEAD (1b) with maleimides 2a-e were examined and the corresponding aza-MBH derivatives $\mathbf{3 a}-\mathbf{j}$ were obtained in moderate to good yields. The results are summarized in table 2 (entries 1-10). Table 2 reveals the minor electronic effect of methyl, phenyl, $p$-Me phenyl and $p$-Br phenyl substituents on maleimide to the yield of the reaction. The structure was further confirmed by X-ray crystallographic analysis of the compound $\mathbf{3 d}$ (figure. 2).

A tentative mechanism is described in figure 3. The reaction is believed to involve the aza-MBH reaction of $\mathbf{1}$ and $\mathbf{2}$ to form the intermediate $\mathbf{4}$ which by the abstraction of proton from the $\alpha$-position of the carbonyl group forms the intermediate $\mathbf{5}$. Subsequent elimination of the catalyst gives the aza-MBH product 3 .

\section{Conclusions}

In summary, we have studied the aza-MBH reaction of diisopropylazodicarboxylate and diethyl azodicarboxylate with maleimides. This reaction conditions have been optimized by varying the catalysts and solvents. Using DABCO as the catalyst under neat condition, the reaction proceeded smoothly and gave the hydrazinesubstituted pyrrolidinone as adduct in good yield. Further transformation of these products is under progress.

\section{Supplementary information}

Crystallographic data for the structure $\mathbf{3 d}$ in this paper have been deposited with the Cambridge Crystallographic Data centre as supplemental publication no. CCDC-779443. Copies of the data can be obtained, free of charge on application to CCDC, 12 Union Road, Cambridge CB2 1EZ, UK (fax: +44 01223336033 or email: deposit@ccdc.cam.ac.uk).

\section{Acknowledgements}

All the authors thank the Council of Scientific and Industrial Research (CSIR), New Delhi, India, for the research fellowship.

\section{References}

1. (a) Morita K 1968 Japan Patent 6803 364; Chem. Abstr. 196869 58828s; (b) Morita K, Suzuki Z and Hirose H 1968 Bull. Chem. Soc. Jpn. 41 2815; (c) Baylis A B and Hillman M E D 1972 Ger. Offen. 2 155 113; 1972 Chem. Abstr. 77 34174; (d) Hillman M E D and Baylis A B 1973 U. S. Patent 3743 669; (e) Basavaiah D, Bhavani A K D, Pandiaraju S and Sarma P K S 1995 Synlett 243; (f) Radha Krishna P, Sachwani R and Srinivas Reddy P 2008 Synlett 2897; (g) Zulykama Y, Uma U, Devi P C and Perumal P T 2009 Can. J. Chem. 87 1682; (h) Saikia M and Sarma J C 2010 Can. J. Chem. 88 1271; (i) Shi M, Wang F-J, Zhao M X and Wei Y (eds) 2011 The chemistry of the Morita-Baylis-Hillman reaction (Cambridge, UK: The Royal Society of Chemistry) p 561; (j) Declerk V, Martinez J and Lamaty F 2009 Chem. Rev. 109 1; (k) Gomez C, Gicquel M, Carry J-C, Schio L, Retailleau P, Voituriez A and Marinetti A 2013 J. Org. Chem. 78 1488; (1) Reddy B V S, Reddy M R, Gopal Rao Y, Yadav J S and Sridhar B 2013 Org. Lett. 15 464; (m) Basavaiah D, Reddy B S and Badsara S S 2010 Chem. Rev. 110 5447; (n) Basavaiah D and Veeraraghavaiah G 2012 Chem. Soc. Rev. 4168

2. (a) Drewes S E and Roo G H P 1988 Tetrahedron 44 4653; (b) Basavaiah D, Rao P D and Hyma R S 1996 Tetrahedron 52 8001; (c) Ciganek E 1997 Org. React. 51 201; (d) Langer P 2000 Angew. Chem., Int. Ed. 39 3049; (e) Basavaiah D, Rao A J and Satyanarayana T 2003 Chem. Rev. 103 811; (f) Ravinder M, Sadhu P S, Santhoshi A, Narender P, Swamy Gundimella Y S K, Ravikumar K and Rao J V 2010 Synthesis 573; (g) Wang Y, Liu L, Wang D and Chen Y-J 2012 Can. J. Chem. 90(1) 85; (h) Shanmugam P and Vaithiyanathan V 2009 Can. J. Chem. 87(4) 591

3. (a) Price K E, Broadwater S J, Jung H M and McQuade D T 2005 Org. Lett. 7 147; (b) Aggarwal V K, Fulford 
S Y and Lloyd-Jones G C 2005 Angew. Chem., Int. Ed. 44 1706; (c) Price K E, Broadwater S J, Walker B J and McQuade D T 2005 J. Org. Chem. 70 3980; (d) Radha Krishna P, Sachwani R and Srinivas R P 2008 Synlett 2897

4. (a) Santos L S, Pavam C H, Almeida W P, Coelho F and Eberlin M N 2004 Angew. Chem., Int. Ed. 43 4330; (b) Krafft M E, Haxell T F N, Seibert K A and Abboud K A 2006 J. Am. Chem. Soc. 128 4174; (c) Gnas Y and Glorius F 2006 Synthesis 1899

5. (a) Iwabuchi Y, Nakatani M, Yokoyama N and Hatakeyama S 1999 J. Am. Chem. Soc. 121 10219; (b) Yang K-S, Lee W-D, Pan J-F and Chen K-M 2003 J. Org. Chem. 68 915; (c) Imbriglio J E, Vasbinder M M and Miller S 2003 J. Org. Lett. 5 3741; (d) McDougal N T and Schaus S E 2003 J. Am. Chem. Soc. 125 12094; (e) Wang J, Li H, Yu X, Zu L and Wang W 2005 Org. Lett. 7 4293; (f) Xu J, Guan Y, Yang S, Ng Y, Peh G and Tan C-H 2006 Chem. Asian J. 1 724; (g) Berkessel A, Roland K and Neud€orfl J M 2006 Org. Lett. 8 4195; (h) Nakano A, Takahashi K, Ishihara J and Hatakeyama S 2006 Org. Lett. 85357

6. (a) Perlmutter P and Teo C C 1984 Tetrahedron Lett. 25 5951; (b) Xu Y-M and Shi M 2004 J. Org. Chem. 69 417; (c) Shi Y-L, Xu Y-M and Shi M 2004 Adv. Synth. Catal. 346 1220; (d) Back T G, Rankic D A, Sorbetti J M and Wullf J E 2005 Org. Lett. 7 2377; (e) Shi Y-L and Shi M 2006 Tetrahedron $\mathbf{6 2} 461$

7. (a) Raheem I T and Jacobsen E N 2005 Adv. Synth. Catal. 347 1701; (b) Buskens P, Klankermayer J and Leitner W 2005 J. Am. Chem. Soc. 12716762

8. (a) Shi M and Xu Y-M 2002 Angew. Chem. Int. Ed. 41 4507; (b) Kawahara S, Nakano A, Esumi T, Iwabuchi Y and Hatakeyama S 2003 Org. Lett. 5 3103; (c) Shi M, Xu Y-M and Shi Y-L 2005 Chem. Eur. J. 11 1794; (d) Matsui K, Takizawa S and Sasai H 2005 J. Am. Chem. Soc. 127 3680; (e) Matsui K, Tanaka K, Horii A, Takizawa S and Sasai H 2006 Tetrahedron: Asymmetry 17 578; (f) Shi M and Chen L-H 2003 Chem. Commun. 1310; (g) Shi M, Chen L-H and Li C-Q 2005 J. Am. Chem. Soc. 127 3790; (h) Shi M and Li C-Q 2005 Tetrahedron: Asymmetry 16 1385; (i) Shi M, Chen L-H and Teng W-D 2005 Adv. Synth. Catal. 347 1781; (j) Liu Y-H, Chen L-H and Shi M 2006 Adv. Synth. Catal. 348 973; (k) Matsui K, Takizawa S and Sasai H 2006 Synlett 761; (1) Gausepohl R, Buskens P, Kleinen J, Bruckmann A, Lehmann C W, Klankermayer J and Leitner W 2006 Angew. Chem., Int. Ed. 453689

9. (a) Takagi M and Yamamoto K 1991 Tetrahedron 47 8869; (b) Shi M and Xu Y-M 2002 Eur. J. Org. Chem. 696; (c) Shi M, Xu Y-M, Zhao G-L and Wu X-F 2002 Eur. J. Org. Chem. 3666; (d) Shi M and Xu Y-M 2003 J. Org. Chem. 68 4784; (e) Shi M and Zhao G-L 2002 Tetrahedron Lett. 434499
10. (a) Kamimura A, Gunjigake $\mathrm{Y}$, Mitsudera $\mathrm{H}$ and Yokoyama S 1998 Tetrahedron Lett. 39 7323; (b) Shi M and Zhao G-L 2004 Tetrahedron 60 2083; (c) Dadwal S, Mobin S M and Namboothiri I N N 2006 Org. Biomol. Chem. 4 2525; (d) Terzidis M A, Tsiaras V G, Stephanidou-Stephanatou J, Tsoleridis C A, Psycharis V and Raptopoulou C P 2012 Synthesis 443392

11. (a) Loh T P, Huang J M, Goh S H and Vittal J J 2000 Org. Lett. 29 1291; (b) Solvent-free organic synthesis Tanaka K (ed) 2003 (Weinheim: Wiley-VCH); (c) Nun P, Martinez J and Lamaty F 2010 Synthesis 2063; (d) Roopan S M, Maiyalagan T and Khan F N 2008 Can. J. Chem. 86 1019; (e) Azizi N, Mehrazma S and Saidi M R 2006 Can. J. Chem. 84800

12. (a) Cave G W W, Raston C L and Scott J L 2001 Chem. Commun. 2159; (b) Tanka K and Toda F 2000 Chem. Rev. 100 1025; (c) Kamal A, Khan M N A, Srikanth Y V V and Reddy K S 2008 Can. J. Chem. 86 1099; (d) Das S, Rahman M, Kundu D, Majee A and Hajra A 2010 Can. J. Chem. 88 150

13. (a) Reddy C R, Reddy M D, Srikanth B and Prasad K R 2011 Org. Biomol. Chem. 9 6027; (b) Basavaiah D, Lenin D V and Veeraraghavaiah G 2011 Curr. Sci. 101 888

14. Acheson R M 1976 An introduction to the chemistry of heterocyclic compounds (New York: John Wiley \& Sons)

15. Badger G M 1961 The chemistry of heterocyclic compounds (New York: Academic Press)

16. Jackson A H 1979 Pyrroles in comprehensive organic chemistry- the synthesis and reaction of organic compounds, Barton D and Ollis W D (eds) chapter 17.1, vol. 4 (Oxford: Pergamon Press) p 275

17. Brower J, Lightner D A and Mc Donagh A F 2001 Tetrahedron 577813

18. (a) Marti C and Carreira E M 2005 J. Am. Chem. Soc. 127 11505; (b) Duvall J R, Wu F and Snider B B 2006 J. Org. Chem. 71 8579; (c) Feldman K S, Nuriye A Y and Li J 2011 J. Org. Chem. 76 5042; (d) Oakdale J S and Boger D L 2010 Org. Lett. 12 1132; (e) Hewlett N M and Tepe J J 2011 Org. Lett. 13 4550; (f) Reddy C R, Reddy M D, Srikanth B and Prasad K R 2011 Org. Biomol. Chem. 96027

19. (a) Karthikeyan K, Seelan T V, Lalitha K G and Perumal P T 2009 Bioorg. Med. Chem. Lett. 19 3370; (b) Praveen C, Karthikeyan K, Perumal P T 2009 Tetrahedron 65 9244; (c) Ramachandiran K, Karthikeyan K, Muralidharan D and Perumal P T 2010 Tetrahedron Lett. 513006

20. Karthikeyan K and Perumal P T 2009 Synlett 2366

21. (a) Zulykama Y and Perumal P T 2007 Aust. J. Chem. 60 205; (b) Zulykama Y and Perumal P T 2009 Tetrahedron Lett. 503892 